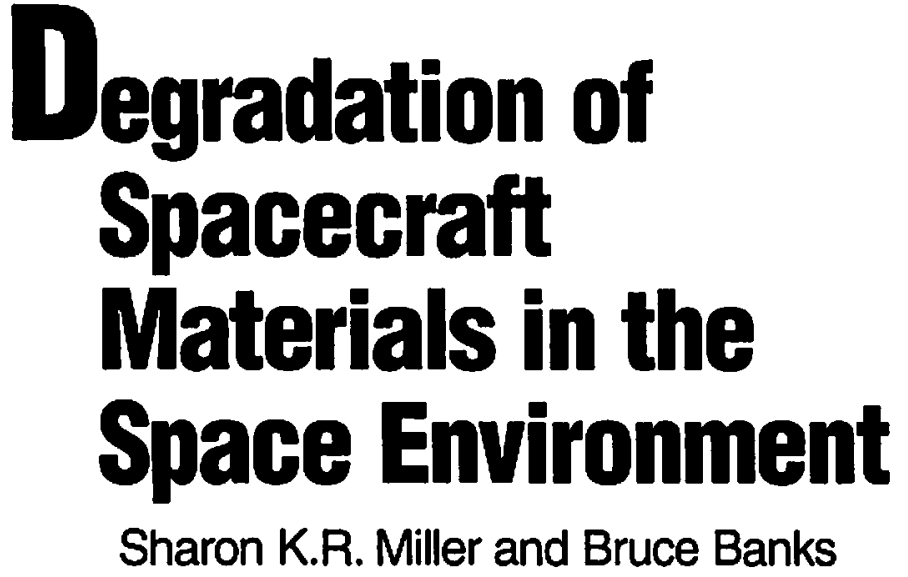

\begin{abstract}
When we think of space, we typically think of a vacuum containing very litile matter that lles between the Earth and other planelary and stellar bodles. However, the space above Earth's breathable atmosphere and beyond contains many things that make designing durable spacecraft a challenge. Depending on where the spacocraft is fying, it may encounter atomic orygen, uttraviolat and other forms of radiation, charged particles. micrometeorodds and debris, and temperature extremes. These emironments on their own and in combination can cause degradation and laibure of polymers, composites, paints and other materials used on the exterlor of spacecraft tor thermal control, structure, and power generation. This articto brielly discusses and glves examples of some of the degradation experienced on spacecraft and flight expertments as a result of the space environment and the use of ground and space data to predict durability.
\end{abstract}

Examples of Degradation from the Intemational Space Station, Low Earth Orbit Flight Experiments, and the Hubble Space Telescope

The ISS was one of the first examples of designing for long-term durability in the low Earth ortit (LEO) environment. Early shuttle missions experienced texturing and thinning of polyimide Kapton and other carbon-containing materials from exposure in LEO. ${ }^{1}$ The primary reason for the degradation was found to be oxidation by the atomic oxygen in LEO.' At the time, polyimide Kapton $\mathrm{H}$, a specific type of Kapton. was under consideration as the prime candidate for the solar array support structure for ISS. Kapton was attractive for this application because the solar cells needed to be bonded to a flexible and strong surface that was lightweight and had the right thermal properties, so the array could be stowed and unfolded like a sail when installed on the ISS. Kapton $\mathrm{H}$ atomic oxygen erosion data from shuttle flight information caused concem for the survivability of Kapton for use as a solar array blanket. ${ }^{2}$ High levels of erosion for Kapton also were observed on the Long Duration Exposure Facility (LDEF) experiment, a free-flying collection of experiments launched from and later retrieved by the Space Shuttle to look at the space environment and its effect on a variety of materials (see the Edwards ct al. article in this issue). Figure 1 shows on-orbit photos of a Kapton blanket-covered panel on LDEF at deployment and after 69 months of space exposure, showing that the outer layer of Kapton on the blanket is missing because it had been totally oxidized into gaseous products. ${ }^{3}$ Malerials that are alscady in their highest oxidation state such as siticon dioxide and aluminum oxide, however, do not react with the atomic oxygen in the LEO environment.' Since Kapion had all of the desired properties for use on ISS other than its interaction with the environment, different types of thin-film metal oxide coatings applied by physical vapor deposition were tried as a protective barrier. Metal oxide coatings proved to be very effective barriers, but where there were defects in the coating caused by surface imperfections or dust particles or scratches from handling postcoating, the atomic oxygen could still reach the Kapton and create areas of undercutting on the surface. ${ }^{5}$ Undercutting, such as that shown in ligure 2 from LDEF, does not pose too much of a problem for maintaining durability unless there are so many defects or scratches that the undercut areas can connect and cause sections of the polymer to be removed or initiate tearing across the surface of the polymer. The array blanket that is on ISS currently is protected with 


\section{Degradation of Spacecraft Materials in the Space Environment}

a thin-film sputter-deposited silicon dioxide coating that has allowed the array to survive longer than the 15-year mission life for which it was originally designed, but it is important to note that there was substantial ground testing and qualification that occurred prior to fabrication to ensure that the number of defects in the coating were low enough such that less than half of the thickness of the array blanket would be removed in onder to enable the blanket to survive for the desired mission lifetime. 6

Unfortunately, not all hardware that is flown undergoes such careful testing. Sometimes assumptions are made that a solution that initially looks good will work without being tested due to a lack of time and funding to verify its performance before flight. An example of this is the solar array blanket box cover on ISS. Much work went into the array blanket itself, but the thermal blanket covering the box in which the array blanket was stowed was not tested in the environment pre-flight. The thermal blanket was composed of polyimide Kapton, which was aluminized on both sides. The assumption was made that since the Kapton already had an aluminum coating, which would serve as a barrier to the atomic oxygen and react with it to make aluminum oxide on the surface, the thermal blanket would not degrade in the environment. Figure 3.3 shows the damage caused by atomic oxygen on the blanket box cover after only a year of exposure on ISS, while the expected lifetime was supposed to have been 15 years. Evaporaled aluminum coatings typically contain more defects than sputterdeposited coatings, but the failune of the blanket box cover was not so much caused by the number of coating defects but by the fact that the Kapton was coaled on both sides. ${ }^{3}$ It was found later through a combination of observation of samples flown on flight experiments and Monte Carlo-based computational modeling that atomic oxygen upon impact with a surface can react, recombine, or bounce off of the surface.s When it bounces off of the surface, the energy of the atomic oxygen is reduced to thermal energy, but it is still very reactive and free to react with another surface. ${ }^{8}$ In the case of the double aluminized Kapton blanket, the atomic oxygen could enter a defect and react with the Kapton until reaching the second aluminum coating underneath. At the surface of the aluminum coating, once the aluminum is oxidized, the atomic oxygen would no longer be able to react with it, so it could only bounce off of the surface to react with the surrounding Kapton or recombine with other oxygen atoms. Essentially, in the case of the ISS blanket

a

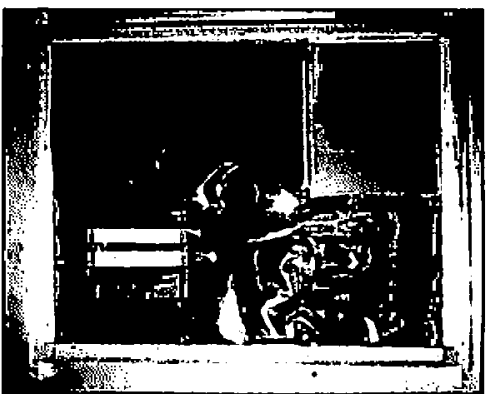

b

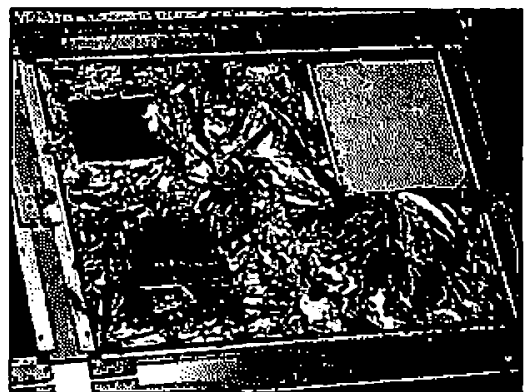

Figure 1. Long Duration Exposure Facility tray F9. (a) Pre-flight photo and (b) on-orbit pholo taken during STS-32, showing complele loss of the outer Kapton layer of the blanket. 10 The aluminized layer is stiil intact.

a

b
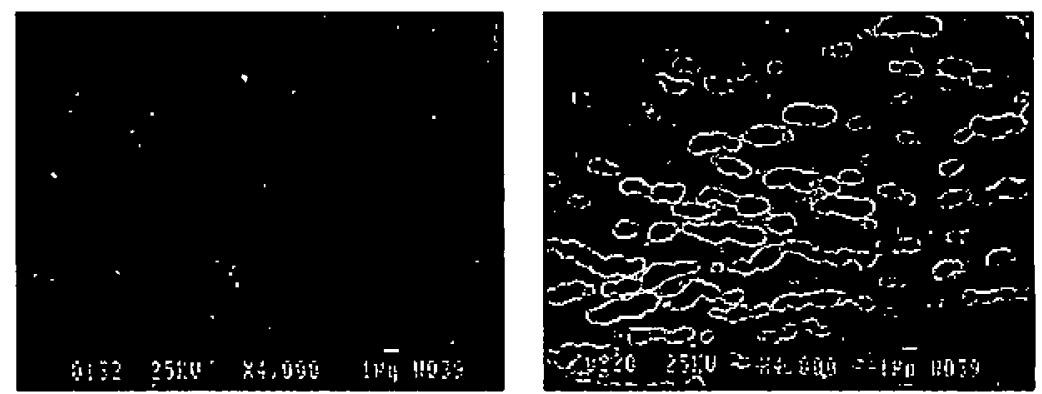

Figure 2. Photos of aluminized Kapion llown on the Long Duration Exposure Facility with the aluminum-coated side tacing the space environment. (a) Delect areas in the aluminum coating and (b) the same sample with the aluminum coating chemically removed to show the undercut cavities under the defect sites causod by atomlc oxygen erosion of the Kapton. ${ }^{19}$

a

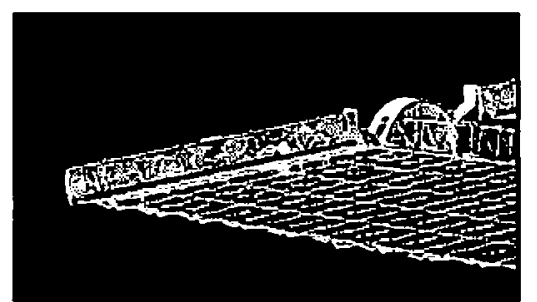

b

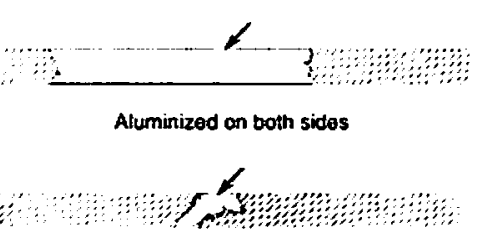

Aluminized on exposed side crily

Figure 3. Damage and damage modeling of the solar array blanket box cover on the International Space Station. (a) Photo showing the damage caused by atomic oxygen on the Kapton (aluminum coating on both sides) blanket box cover after one year in orbit. (b) Monte

Carlo modeling comparison of undercutting patterns for Kapton coated with aluminum on both sides compared to a single layer aluminum protective coating on Kapton.

box cover, the atomic oxygen became trapped between the two layers of aluminum causing greater opportunity for erosion of the Kapton between the aluminum layers. The erosion from multiple bounce, trapped atomic oxygen eventually resulted in the removal of most of the Kapton to create two free-standing aluminum films. ${ }^{8}$ If the aluminum barrier had been only on the space-facing side of the 
blanket, the atomic oxygen would have reacted at defect sites to create a narrow channel to the back side and then would have been able to scatter through the open pore polyimide underneath rather than being trapped in the Kapton blanket where it could neact further with the polymer. ${ }^{8}$ This is illustrated by the Monte Carlo atomic oxygen undercutting modeling results for both double-coated and singlecoated Kapton shown in Figure 3b." Pre flight qualification testing could have avoided this degradation problem.

Not all testing, however, is straightforward. Unfortunately, there is no easy way to exactly replicate the environment of space on Earth. There are physical limitations as to what can be done to generate atomic oxygen, vacuum ultraviolet (VUV) (100-200 nm) and near UV (200-400 nm) radiation, ionizing radiation, and thermal extremes in the exact conditions and pro portions as in space. So flight experiments, such as those flown on the Space Shuttle, on free carriers such as LDEF, 10 and on the outside of ISS," are used to provide material degradation information that can be used to help correlate what is happening in space with what is occurring in groundbased testing facilities. One of the experiments on the Materials Intemational Space Station Experiment (MISSE) 2 and 4 was designed to measure the erosion of a coated polyimide in space, compared to its erosion in a ground-based test facility to develop a cormelation factor that could be used to mone neliably predict the in-space durability of coated polymers tested in a ground. based system." The correlation factor that was determined $(\sim 18$ times more erosion for coated Kapton in a ground plasma system than in LEO) along with ground system testing of some of the Kapton multilayer insulation (MLI) blanket from HST was used to predict the condition of the MLI for Servicing Mission 4 (SM4)." The prediction that the MLI would not be degraded to the extent that it would need to be covered by the astronauts during HST SM4 allowed this task to be moved to the end of the schedule and listed as optional, thereby freeing time for other more critical tasks."I It was observed on SM4 that the condition of the Kapton MLJ was acceptable, so the task to cover this bay was performed as one of the last servicing tasks. Another experiment flown on MISSE was designed to measure the erosion rates of 40 different polymers and graphite to gain better understanding of polymer erosion in LEO and to develop a predictive tool to enable estimation of the erosion of new polymers under consideration for use in space, based on knowing the chemical structure, density, and ash content deter- mined in a ground-based atomic oxygen exposure facility"1.12 Ash content is important in the prediction because the ash present in a material is the part that cannot be oxidized and can accumulate on the surface during exposure shiclding the underlying material from further oxidation. In essence, it develops its own protective coating that can make the observed erosion lower than what would be expected based on the erosion of the bulk material. Because most of the damage observed in LEO is from atomic oxygen attack, most of the work to date has focused on atomic oxygen erosion. It is still not totally understood what role the other environments play in material degradation, as some components of the environment appear to play a synergistic role in degradation for some materials but not for others.

A good example of synergistic environment effects is the severe on-orbit cracking of the outer layer of the vapor-deposited aluminum-coated fuorinated ethylene propylene (FEP) Teflon MLJ blanket observed on the solar-facing side of the HST during the second servicing mission. ${ }^{13}$ Higure 4 shows the embrittled MLI blanket after 6.8 years in space. A roview boand that investigated the severe FEP degradation on HST concluded that electron and proton radiation combined with on-orbit thermal cycling caused the observed cracking of the MLI at stresconcentrated locations and that damage increased with the combined total dose of ionizing radiation, ultraviolet radiation, and $x$-rays with thermal cycling. ${ }^{33}$ The conclusion was that radiation-induced chain scission was the primary mechanism of degradation, and the damage rate was significantly affected by on-orbit temperature. Although damage was observed in accelerated ground-based exposures, it did not simulate the extent of damage observed on HST. ${ }^{13}$ Calibration of ground-based accelerated exposure using space data is needed to obtain more accurate simulation of this effect of the environment.

Another example of a combined environmental effect on materials, first observed on LDEF, was the formation of a darkened contaminant layer on surfaces due to atomic oxygen and VUV radiation." Figure 5 shows a close-up of a tray of materials that was $38^{\circ}$ from the ram (or orbital) direction on LDEF on the Solar-Array Materials Passive experiment." The light-coloned samples (rectangular, square, and tensile shaped) are silicones that contained a lot of volatiles that, when in space, produced large quantities of contaminants on neighboring surfaces and samples. A combination

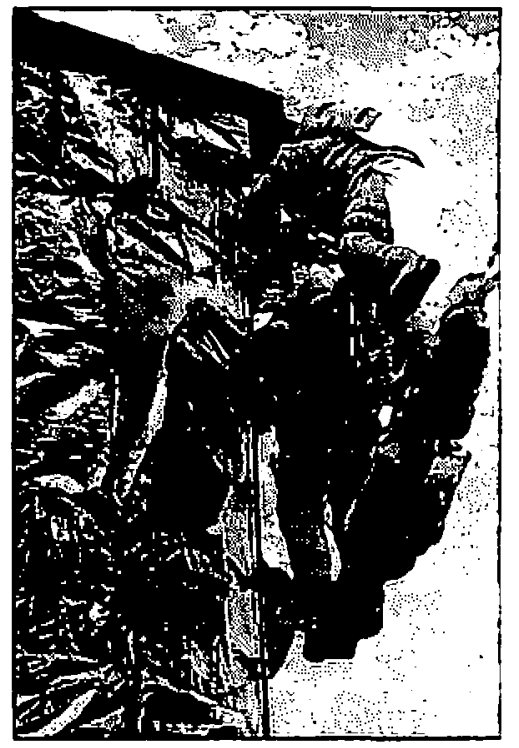

Figure 4. Phalograph of the Hubble Space Telescope on orbil during the second servicing mission showing cracking and tearing of the multilayer insulation after 6.8 years of spaco exposure due to what is believed to be a combination of ionizing radiation. ultraviofet radiation, $x$-rays, and thermal cycling.

of VUV bond breakage of the deposited volatile silicone fragments, hydrocarbon deposition, and atomic oxygen conversion of the contaminant surface layer to silica allowed the build-up of a darkened contaminant layer. There is a light-colored "shadowed" region directly to the right of the samples. This is where atomic oxygen arrival was shielded, and hence the deposited contaminant fragments were able to re-evaporate. Because a darkened contaminant layer can lead to significant changes in optical and thermal properties of the material on which it is deposited, silicones used in space cursently have to meet stricter guidelines with regard to the volatile condensable products that they give off during heating and under vacuum so as not to cause significant power losses, changes in performance of optics, or overheating.

Combined environments of atomic oxygen and micrometeoroid and debris impacts also can lead to greater damage than by either alone. Figure tis shows a photo of a silver-FEP Teflon blanket flown on the ram-facing side of LDEF, showing 


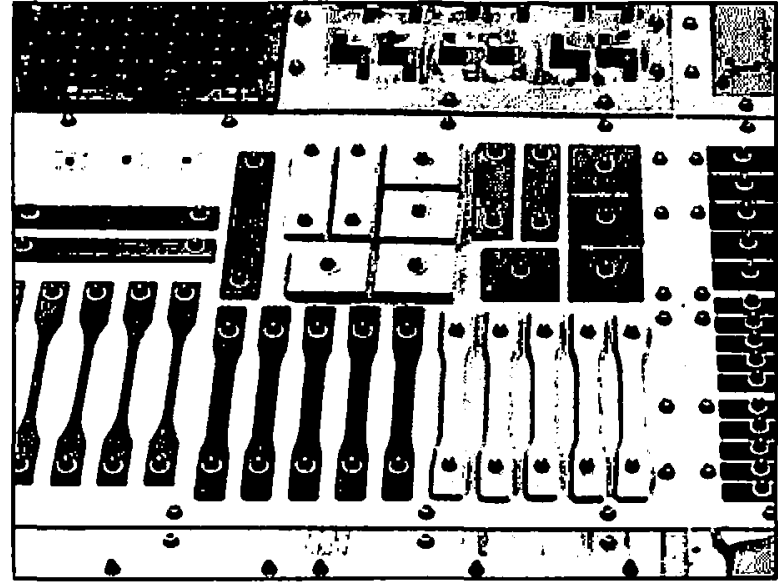

Figure 5. Section of the Long Duration Exposure Facility Solar Array Materials Passive Experiment (tray A8) showing dark contamination to the right of the silicone (light gold colored) rectangular and dog bone-shaped samples.

a
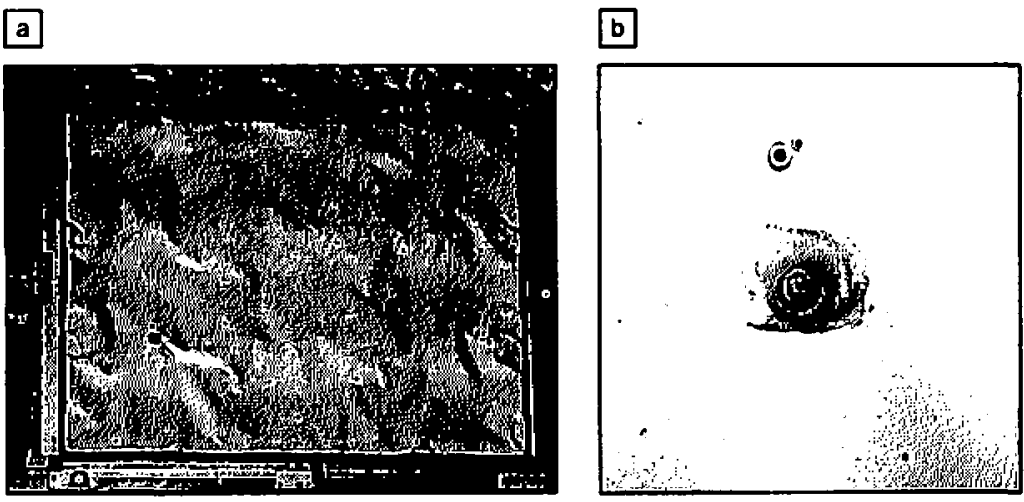

Figure 6. Long Duration Exposure Facility tray A10 showing micrometeoroid and debris impacts. (a) On-orbit photo of tray A10 and (b) post-flight close-up photo of the impact site showing the impact hole, delamination area, and the darkened rings caused by oxidation of the siver.

micromeleoroid and debris impacts. The impacts were found to cause delamination of the silver-FEP Teflon composite, which then allowed atomic oxygen to enter the impact hole to oxidize the underlying exposed silver. ${ }^{15}$ This resulted in the darkened rings observed around the impact holes. An example is shown in the close-up image in Figure 6b.15 LDEF provided a large collection of impact data that covened a wide range of impact craters from below $0.01 \mathrm{~mm}(10 \mu \mathrm{m})$ to $5.25 \mathrm{~mm}$.6.17 Figurn! 7 provides an example of an impact in alumigold (a chromatc conversion coating) on aluminum from LDEF. When micrometeoroids and debris impact spacecraft surfaces, they generally vaporize along with some of the material they impact, which then can condense on the surface. This condensate and the condensed molten material created by the impact form a wave or splash pattem around the crater. Fortunately, the size distribution and number ane currently low enough that the proper design (multiple spaced layers for piping, multilayer shielding, or redundant systems) can reduce the damage that impacts can cause. ${ }^{13}$

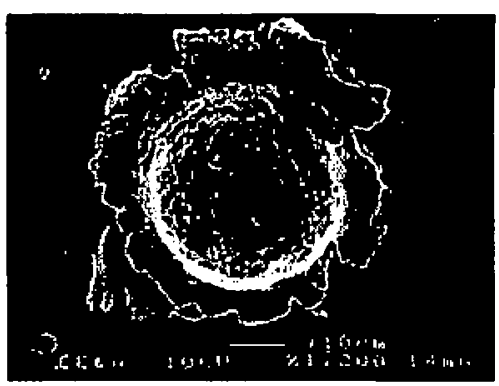

Figure 7. Impact crater on alumigold (chromate conversion coating) on aluminum irom the Long Duration Exposure Facility.

\section{Conclusions}

This article provided a few examples of degradation that have been observed on spacecraft as a result of the Earth orbital environment. The experience gained through observing damage on spacecraft and verification of the causes of damage through a combination of spaceflight experiment, ground-based testing, and modeling has led to better spacecraft design. In spite of the wealth of knowledge that has been gained, there are still many unknowns with regard to the role each environment plays in the damage and in the presence of, or lack of, synergistic effects with other components of the environment. What has been learned, however, has resulted in better methods of testing, evaluation, and analysis that are not only valuable for enabling design of more durable spacecraft for the Earth's orbital environment but also may provide guidance for durable spacecraft design for other environments beyond Earth.

\section{Acknowledgments}

The authors would like to thank Kim de Groh of NASA GRC for providing useful references and information for this article.

\section{References}

1. L.J. Leger, Oxygen Atom Reaction with Shuttle Materials at Orbital Altitudes. NASA TM-58246 (1982).

2. A.F. Whitaker, paper presented at the AlAA Shutle Environment and Operations Meeting. Washington, DC, 31 October-2 November 1983, AlAA-83-2632-CP.

3. R.L. O'Neal, A.S. Levine, C.C. Kiser, Photographic Survey of the LDEF Mission, NASA SP 531 (1996).

4. B.A. Banks, M.J. Mirtich, S.K. Rutledge, D.M. Swec, H.K. Nahra, 23rd Aerospace Sciences 
Meeting, Reno, NV, 14-17 January 1985, NASA TM-87051.

5. B.A. Banks, B.M. Auer, S.K. Rutledge, C.M Hill, Proceedings of the 4th Ammual Workshop on Space Operations Automation and Robotic (SOAR 90), Albuquerque, NM, 26-28 June 1990. 6. S.K. Rutledge, R.M. Olle, Proceedings of the 38th International SAMPE Symposium, 10-13 May 1993, pp. 679-693.

7. M.J. Forkapa, C.R. Stidham, B.A. Banks, S.K Rutledge, D.H. Ma, E.A. Sechkar. Third International Conference on Protection of Materials and Structures from the Low Earth Orbit Space Environment, Toronto, Canada. 25-26 April 1996, NASA TM-107212.

8. B.A. Banks, R. Demko, Atomic Orygen Protection of Materials in Low Earth Orbit, NASA TM-2002-211360 (February 2002).

9. B.A. Banks, K.K. de Groh, S.K. Rutledge, C.A. Haytas, 44th Annual Meeting by the Intermational Society of Optical Engineering Denver, CO, 21 July 1999, NASA TM-1999. 209179.
10. LDEF-69 Months in Space. First Post-Rotrieual Symposium, A.S. Levine, Ed., NASA Conference Publication 3134, Parts 1-3, Kissimmee, F., 2-8 June 1991.

11. K.K. de Groh, B.A. Banks, J.A. Dever, D.A. Jaworske, S.K. Miller, E.A. Sechkar, S.R. Panko, NASA Glenn Research Center's Materials International Space Station Experiments (MISSE 1-7), Interntational Symposium on SM/MPAC and SEED Experintents (JAXA), Epochal Tsukuba Japan, 10-11 March 2008, NASA TM-2008215482.

12. B.A. Banks, J.A. Backus, M.V. Manno, D.I Waters, K.C. Cameron, K.K de Groh, in Atomic Oxyeren Erosion Yield Prediction for Spacecraf Polymers in Low Earth Orbit, 11 th Intemational Symposium on Materials in the Space Environment (ISMSE-11), 15-18 September 2009.

13. J. Dever, B. Banks, K. de Groh, S. Miller, in Handbook of Environmental Degradation of Materinls, M. Kutz, Ed. (William Andrew Publishing, Norwich, NY, 2005), pp. 465-501.
14. B.A. Banks, J.A. Dever, L. Gebauer, C.M Hill, in Proceedings of the LDEF-69 Months in Space, First Post-Retrizal Symposium, NASACP. 3134, Part 2, 2-8 June 1991, pp. 801-815.

15. H.G. Pippin, Analysis of Silverized Teflon Thermal Control Material Flown on the Long Duration Exposure Facility, NASA CR-4663 (July 1995).

16. E.M. Silverman, Space Environmental Effects on Spacecraft: L.EO Materials Selection Guide. NASA CR-1661 (August 1995).

17. T. See, M. Allbrooks, D. Atkinson, C. Simon M. Zolensky, "Meteoroid and Debris Impact Features Documented on the Long Duration Exposure Facility," (Preliminary Report Compiled by Members of the LDEF Meteomid and Debris Special Investigation Group, NASA JSC 24608, August 1990).

18. R.L. O'Neal A.S Levine, C.C. Kiser Photographic Surocy of the LDEF Mission. NASA SP-531 (1996).

19. K.K. de Groh, B.A. Banks, J. Spacecr. Rockets 31 (4), 656 (1994) 